\title{
Comment on "Diffusion Monte Carlo study of jellium surfaces: Electronic densities and pair correlation functions"
}

\author{
J. M. Pitarke \\ Materia Kondentsatuaren Fisika Saila, Zientzi Fakultatea, Euskal Herriko Unibertsitatea, \\ 644 Posta Kutxatila, E-48080 Bilbo, Basque Country, Spain \\ and Donostia International Physics Center (DIPC) and Centro Mixto CSIC-UPV/EHU, Manuel de Lardizabal Pasealekua, \\ E-20018 Donostia, Basque Country, Spain \\ (Received 11 August 2003; revised manuscript received 27 January 2004; published 24 August 2004)
}

\begin{abstract}
In a fixed-node diffusion Monte Carlo calculation of the total energy of jellium slabs, Acioli and Ceperley [Phys. Rev. B 54, 17199 (1996)] reported jellium surface energies that at low electron densities were significantly higher than those predicted in the local-density approximation (LDA) of density-functional theory. Assuming that the fixed-node error in the slab and the bulk calculations cancel out, we show that their data yield surface energies that are considerably closer to the LDA and in reasonable agreement with those obtained in the random-phase approximation.
\end{abstract}

DOI: $10.1103 /$ PhysRevB.70.087401

PACS number(s): 71.10.Ca, 71.15.Mb, 71.45.Gm

Acioli and Ceperley ${ }^{1}$ presented the results of fixed-node difussion Monte Carlo (DMC) calculations of the total energy of jellium slabs at five different electron densities. Assuming that the released-node correction is very small at the electron densities of interest, these authors extracted surface energies by substracting from the fixed-node slab energy the corresponding released-node bulk energies of Ceperley and Alder, ${ }^{2}$ as parametrized by Perdew and Zunger. ${ }^{3}$ They concluded that at low electron densities jellium surface energies are significantly higher than those predicted in the localdensity approximation (LDA) of density-functional theory (DFT).

In this Comment, we show that combining fixed-node slab and release-node bulk energies results in substantial imprecision. Instead, we expect a large degree of cancellation between the fixed-node error in the slab and the bulk calculations, and conclude that by substracting from the fixednode slab energies the corresponding fixed-node bulk energies one obtains jellium surface energies that are considerably closer to the LDA (Ref. 4) and in reasonable agreement with those obtained in the random-phase approximation (RPA). ${ }^{5}$

Acioli and Ceperley ${ }^{1}$ considered jellium slabs of density $n_{0}=3 / 4 \pi\left(r_{s} a_{0}\right)^{3}$ ( $a_{0}$ is the Bohr radius), the thickness of the positive background in each slab being $L=7.21\left(r_{s} a_{0}\right)$. Surface energies were then obtained from

$$
\sigma=\left[\varepsilon^{\text {slab }}-\varepsilon^{\text {bulk }}\right] \frac{n_{0} L}{2}
$$

where $\varepsilon^{\text {slab }}$ and $\varepsilon^{\text {bulk }}$ represent slab and bulk energies per particle, respectively. Fixed-node energies per particle in bulk jellium were reported by Ceperley $^{6}$ to be higher than their release-node counterparts by $9 \times 10^{-4}$ Ry for $r_{s}=2$ and $2 \times 10^{-4}$ Ry for $r_{s}=5$. Hence, combining fixed-node slab and release-node bulk energies Eq. (1) yields for $r_{s}=2$ and 5 surface energies that are too large by $\sim 150$ and $5 \mathrm{erg} / \mathrm{cm}^{2}$, respectively. These fixed-node errors represent $\sim 40 \%$ and $\sim 20 \%$ of the LDA correlation energy for $r_{s}=2$ and 5, respec- tively. Furthermore, they are comparable to the deviation of the total surface energies reported in Ref. 1 from those obtained in the LDA.

In order to derive reliable surface energies from the fixednode slab calculations of Ref. 1, we require fixed-node energies of bulk jellium. Such calculations were reported by Ceperley ${ }^{6}$ and more recently by Kwon et al. ${ }^{7}$ for $r_{s}=1,5,10$, and 20. The fixed-node Slater-Jastrow correlation energies of Kwon et al. $^{7}$ can be parametrized in Perdew-Zunger ${ }^{3}$ form, as follows: ${ }^{8}$

$$
\varepsilon_{c}^{\text {bulk }}=\frac{-0.32172}{1+1.3606 \sqrt{r_{s}}+0.3391 r_{s}} \mathrm{Ry} \quad\left(r_{s} \geqslant 1\right),
$$

or in Perdew-Wang ${ }^{9}$ form, as follows;

$$
\varepsilon_{c}^{\text {bulk }}=-\left(0.12436+0.027404 r_{s}\right) \ln \left(1+\frac{16.082}{\alpha}\right) \mathrm{Ry},
$$

with

$$
\alpha=7.5957 r_{s}^{1 / 2}+3.5876 r_{s}+1.8207 r_{s}^{3 / 2}+0.47746 r_{s}^{2} .
$$

Both Eqs. (2) and (3) have been adjusted to fit the fixed-node correlation energies of Kwon et al. ${ }^{7}$ for $r_{s}=1,5$, and 10. The fixed-node bulk energy $\varepsilon^{\text {bulk }}=-0.01482$ Ry obtained by Ceperley and Alder ${ }^{16}$ for $r_{s}=2.07$ is underestimated by Eq. (2) by $2 \times 10^{-5}$ Ry and overestimated by Eq. (3) by $2 \times 10^{-4}$ Ry. At $r_{s}=20$, Eqs. (2) and (3) underestimate the fixed-node bulk energy of Kwon et al. ${ }^{7}$ by $2 \times 10^{-4} \mathrm{Ry}$ and $10^{-4} \mathrm{Ry}$, respectively.

Adding to the correlation energy of Eqs. (2) and (3) the well-known kinetic and exchange energies of a uniform electron gas ${ }^{10}$ and combining these energies with the fixed-node slab energies of Acioli and Ceperley, ${ }^{1}$ Eq. (1) yields the surface energies $\left(\sigma_{1}\right.$ and $\left.\sigma_{2}\right)$ shown in Table I. Also shown in this table are the surface energies reported in Ref. $1\left(\sigma_{\mathrm{AC}}\right)$, together with the LDA and RPA surface energies that we 
TABLE I. Surface energies of jellium slabs with $L$ $=7.21\left(r_{s} a_{0}\right)$, as obtained from Eq. (1) by combining the AcioliCeperley fixed-node slab energies with the fixed-node bulk energies of either Eq. (2) $\left(\sigma_{1}\right)$ or Eq. (3) $\left(\sigma_{2}\right)$, and by combining the AcioliCeperley fixed-node slab energies with release-node bulk energies $\left(\sigma_{\mathrm{AC}}\right)$. At $r_{s}=2.07$, combining the Acioli-Ceperley fixed-node slab energy with the corresponding fixed-node bulk energy $\varepsilon^{\text {bulk }}$ $=-0.01482$ Ry of Ceperley and Alder (Ref. 16) yields $\sigma=-558$ $\mathrm{erg} / \mathrm{cm}^{2} . \sigma_{\mathrm{LDA}}$ and $\sigma_{\mathrm{RPA}}$ represent LDA and RPA surface energies. $\sigma_{0}=\sigma_{s}+\sigma_{\text {es }}+\sigma_{x}$ represents the combined kinetic $\left(\sigma_{s}\right)$, electrostatic $\left(\sigma_{\mathrm{es}}\right)$, and exchange $\left(\sigma_{x}\right)$ contributions to the total surface energy $\sigma$. The correlation surface energy is simply $\sigma_{c}=\sigma-\sigma_{0}$. Units are $\mathrm{erg} / \mathrm{cm}^{2}\left(1 \mathrm{erg} / \mathrm{cm}^{2}=6.2415 \times 10^{-5} \mathrm{eV} / \AA^{2}\right)$.

\begin{tabular}{ccccccc}
\hline \hline$r_{s}$ & $\sigma_{0}$ & $\sigma_{1}$ & $\sigma_{2}$ & $\sigma_{\mathrm{AC}}$ & $\sigma_{\mathrm{LDA}}$ & $\sigma_{\mathrm{RPA}}$ \\
\hline 1.87 & -2402 & -1197 & -1247 & -1035 & -1557 & -1424 \\
2.07 & -1234 & -554 & -592 & -416 & -600 & -497 \\
2.66 & -131 & 242 & 226 & 327 & 180 & 233 \\
3.25 & 52 & 312 & 305 & 365 & 227 & 258 \\
3.93 & 72 & 251 & 249 & 284 & 173 & 191 \\
\hline \hline
\end{tabular}

have obtained for jellium slabs with $L=7.21\left(r_{s} a_{0}\right)$. Small differences of no more than $10 \mathrm{erg} / \mathrm{cm}^{2}$ between these LDA and RPA surface energies and those reported before for the semi-infinite jellium ${ }^{4,5}$ are due to the finite size of our jellium slabs.

An inspection of Table I shows that using in Eq. (1) the fixed-node bulk energies of either Eq. (2) or Eq. (3) brings the DMC surface energies closer to the LDA and to reasonable agreement with the RPA. This is consistent with recent work, where it was shown that upon surface formation there is a persistent cancellation of short-range correlation effects beyond the RPA. ${ }^{11}$ Other approaches have also led to the conclusion that the actual jellium surface energies should be only slightly higher than those obtained in the LDA. ${ }^{12-14}$

Li et al. ${ }^{15}$ calculated the fixed-node DMC surface energy of a jellium slab with $r_{s}=2.07$, the thickness of the positive background being $L=8.52\left(r_{s} a_{0}\right)$. These authors, ${ }^{15}$ unlike Acioli and Ceperley, ${ }^{1}$ extracted from their fixed-node slab energy the corresponding fixed-node bulk energy $\left(\varepsilon^{\text {bulk }}=\right.$ $-0.01482 \mathrm{Ry}),{ }^{16}$ and found $\sigma=-465 \mathrm{erg} / \mathrm{cm}^{2} ;{ }^{17}$ they also performed LDA calculations and found a LDA surface energy of $-567 \mathrm{erg} / \mathrm{cm}^{2}$. We have calculated the LDA and RPA surface energies of a jellium slab with $r_{s}=2.07$ and $L$ $=8.52\left(r_{s} a_{0}\right)$, and have found $\sigma_{\mathrm{RPA}}=-485 \mathrm{erg} / \mathrm{cm}^{2}$ and $\sigma_{\mathrm{LDA}}=-589 \mathrm{erg} / \mathrm{cm}^{2},{ }^{18}$ which are both only $\sim 20 \mathrm{erg} / \mathrm{cm}^{2}$ smaller than the corresponding DMC and LDA surface energies reported in Ref. 15.

Figure 1 exhibits the surface energies of Table I, as a function of $r_{s}$. At the highest densities the agreement between DMC and RPA surface energies is good. At the smallest densities $\left(r_{s} \geqslant 3.25\right)$ the corrected DMC surface energies are still larger than their RPA counterparts. Nevertheless, we note that DMC surface energies are very sensitive to small uncertainties in the parametrization of the bulk energy. Therefore, if one is to quantitatively account for jellium surface energies, both DMC slab and bulk energies entering Eq. (1) should be computed on the same footing.

Small differences between the DMC and RPA calculations come from the correlation contribution to the surface energy. We have carried out calculations of exact kinetic $\left(\sigma_{s}\right)$, electrostatic $\left(\sigma_{\text {es }}\right)$, and exchange $\left(\sigma_{x}\right)$ surface energies for jellium slabs with $L=7.21\left(r_{s} a_{0}\right)$, and we have defined the correlation contribution to the DMC surface energy as

$$
\sigma_{c}=\sigma-\left(\sigma_{s}+\sigma_{\mathrm{es}}+\sigma_{x}\right)
$$

The results we have obtained from our analysis of the AcioliCeperley DMC slab energies are shown in Fig. 2 (see also Table I), together with the LDA and RPA correlation energies that we have obtained for the same jellium slabs. This figure clearly shows that combining fixed-node slab energies with their fixed-node bulk counterparts yields DMC correlation surface energies in reasonable agreement with the results obtained in the RPA. While the LDA underestimates considerably the correlation surface energy for all electron densities, it overestimates the exact exchange surface energy. Large and opposite separate corrections to the LDA for exchange and correlation largely compensate, as discussed before, ${ }^{5,19}$

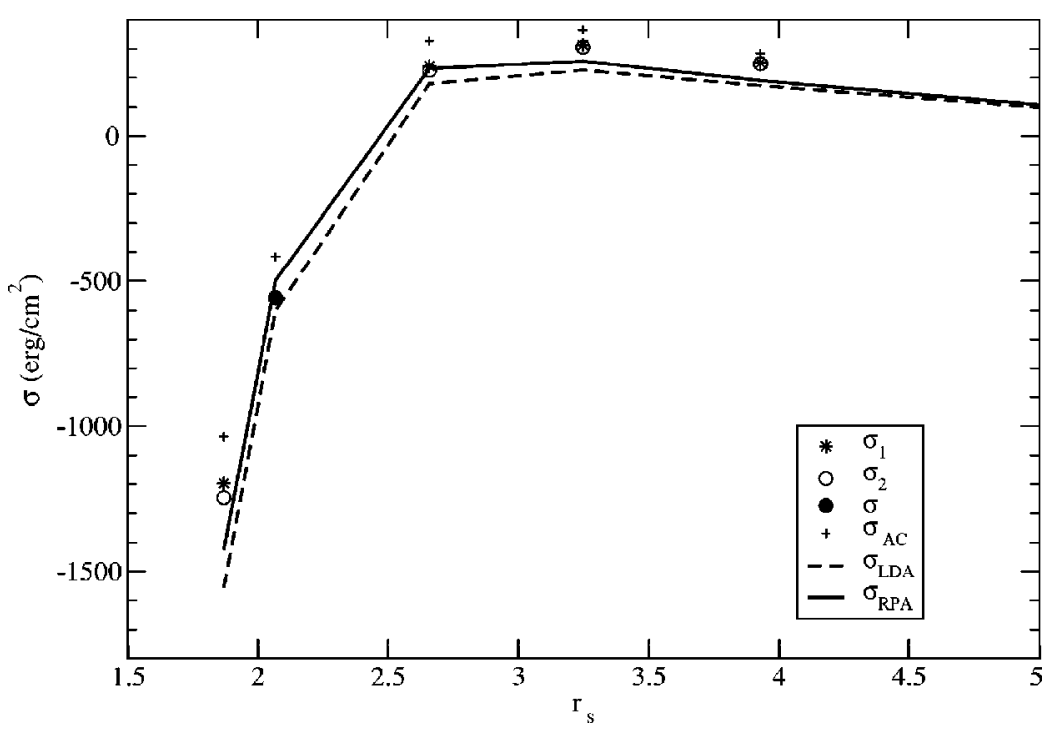

$087401-2$
FIG. 1. Surface energies of jellium slabs with $L=7.21\left(r_{s} a_{0}\right)$, as obtained from Eq. (1). Stars and open circles represent DMC surface energies obtained by combining the Acioli-Ceperley fixednode slab energies with the fixed-node bulk energies of Eqs. (2) and (3), respectively. The solid circle represents the DMC surface energy obtained at $r_{s}=2.07$ by combining the AcioliCeperley fixed-node slab energy with the corresponding fixed-node bulk energy $\varepsilon^{\text {bulk }}$ $=-0.01482$ Ry of Ceperley and Alder (Ref. 16). Crosses represent the surface energies obtained in Ref. 1 by combining fixed-node slab and releasenode bulk energies. Dashed and solid lines represent LDA and RPA calculations, respectively. 


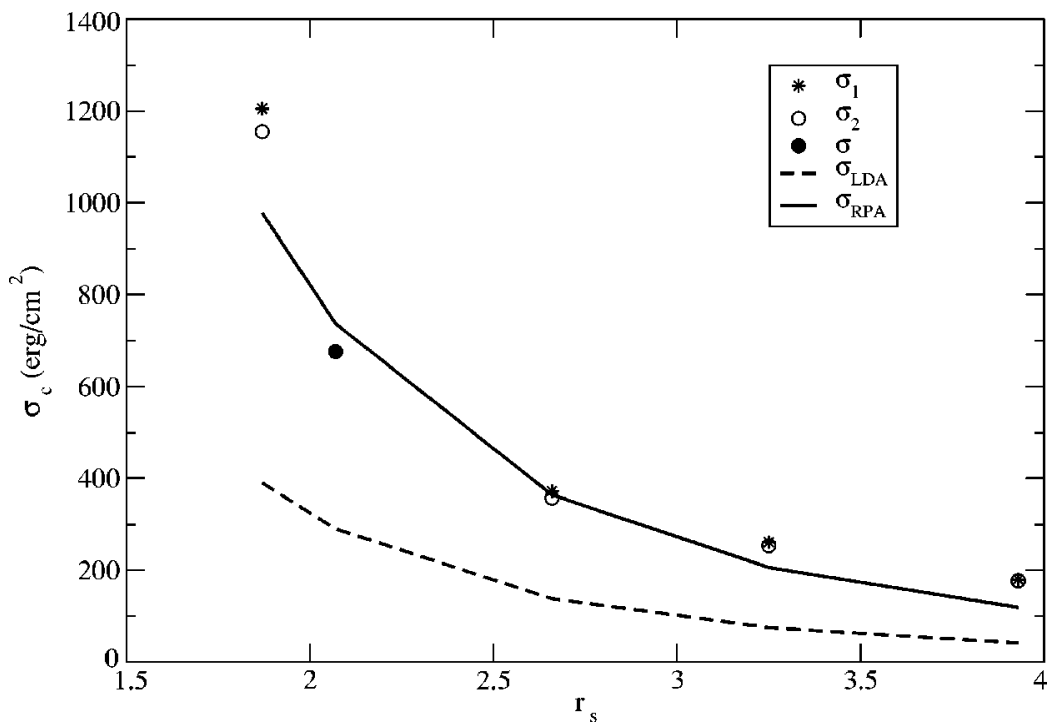

FIG. 2. Correlation contribution to the surface energy. Stars and open circles represent DMC correlation surface energies obtained by combining the Acioli-Ceperley fixed-node slab energies with the fixed-node bulk energies of Eqs. (2) and (3), respectively. The solid circle represents the DMC correlation surface energy obtained at $r_{s}$ $=2.07$ by combining the Acioli-Ceperley fixednode slab energy with the corresponding fixednode bulk energy $\varepsilon^{\text {bulk }}=-0.01482$ Ry of Ceperley and Alder (Ref. 16). Dashed and solid lines represent LDA and RPA calculations, respectively.

and yield LDA surface energies that are close to their nonlocal counterparts.

In conclusion, assuming that the fixed-node error in the slab and the bulk calculations cancel out, the DMC data reported in Ref. 1 yields surface energies that are considerably closer to the LDA and in reasonable agreement with those obtained in the RPA. Nevertheless, at the smallest densities the corrected DMC surface energies are still larger than their RPA counterparts; at these densities, they are also larger than the jellium surface energies extracted from DMC calculations for jellium spheres, ${ }^{20}$ which are found to be close to the
LDA. ${ }^{14}$ Surface energies are extremely sensitive to little uncertainties in both slab and bulk energies; hence, in order to quantitatively account for the impact of nonlocal xc effects on the surface energy one needs to pursue DMC evaluations of both slab and bulk energies on the same footing.

Partial support by the University of the Basque Country, the Basque Unibertsitate eta Ikerketa Saila, and the Spanish MCyT is acknowledged. I thank J. P. Perdew for stimulating discussions and D. M. Ceperley for correspondence related to the unpublished uniform-gas fixed-node data for $r_{s}=2.07$.
${ }^{1}$ P. H. Acioli and D. M. Ceperley, Phys. Rev. B 54, 17199 (1996).

${ }^{2}$ D. M. Ceperley and B. J. Alder, Phys. Rev. Lett. 45, 566 (1980).

${ }^{3}$ J. P. Perdew and A. Zunger, Phys. Rev. B 23, 5048 (1981).

${ }^{4}$ See, e.g., Z. Y. Zhang, D. C. Langreth, and J. P. Perdew, Phys. Rev. B 41, 5674 (1990).

${ }^{5}$ J. M. Pitarke, and A. G. Eguiluz, Phys. Rev. B 57, 6329 (1998); 63, 045116 (2001).

${ }^{6}$ D. M. Ceperley, in Recent Progress in Many Body Theories, Lecture Notes in Physics, edited by J. G. Zabolitzky (SpringerVerlag, Berlin, 1981), Vol. 142.

${ }^{7}$ Y. Kwon, D. M. Ceperley, and R. M. Martin, Phys. Rev. B 58, 6800 (1998).

${ }^{8}$ J. P. Perdew (unpublished).

${ }^{9}$ J. P. Perdew and Y. Wang, Phys. Rev. B 45, 13244 (1992).

${ }^{10}$ D. Pines and P. Nozieres, The Theory of Quantum Liquids (Addison-Wesley, New York, 1989), Vol. I, p. 274.

${ }^{11}$ J. M. Pitarke and J. P. Perdew, Phys. Rev. B 67, 045101 (2003).

${ }^{12}$ Z. Yan, J. P. Perdew, S. Kurth, C. Fiolhais, and L. Almeida, Phys. Rev. B 61, 2595 (2000).

${ }^{13}$ Z. Yan, J. P. Perdew, and S. Kurth, Phys. Rev. B 61, 16430
(2000).

${ }^{14}$ L. M. Almeida, J. P. Perdew, and C. Fiolhais, Phys. Rev. B 66, 075115 (2002).

${ }^{15}$ X.-P. Li, R. J. Needs, R. M. Martin, and D. M. Ceperley, Phys. Rev. B 45, 6124 (1992).

${ }^{16}$ D. M. Ceperley and B. J. Alder (unpublished).

${ }^{17}$ The difference between this surface energy and the surface energy of $-558 \mathrm{erg} / \mathrm{cm}^{2}$ that we have obtained from the AcioliCeperley slab energy (see caption of Table I) is partially due to the difference in the thickness of the jellium slabs considered in Refs. 1 and 15.

${ }^{18}$ Due to oscillatory quantum-size effects, differences between the finite-slab surface energies and those reported before for the semi-infinite jellium (Ref. 4 and 5) are larger for $L$ $=8.52\left(r_{s} a_{0}\right)$ than in the case of $L=7.21\left(r_{s} a_{0}\right)$.

${ }^{19}$ N. D. Lang and L. J. Sham, Solid State Commun. 17, 581 (1975); D. C. Langreth and J. P. Perdew, ibid. 17, 1425 (1975); Phys. Rev. B 15, 2884 (1977).

${ }^{20}$ F. Sottile and P. Ballone, Phys. Rev. B 64, 045105 (2001). 